\title{
Building integral projection models with non-independent vital rates
}

\author{
Yik Leung Fung ${ }^{1}$, Ken Newman ${ }^{2}$, Ruth King ${ }^{1}$, and Perry de Valpine ${ }^{3}$ \\ ${ }^{1}$ University of Edinburgh \\ ${ }^{2}$ The University of Edinburgh School of Mathematics \\ ${ }^{3}$ University of California, Berkeley
}

November 26, 2021

\begin{abstract}
Population dynamics are functions of several demographic processes including survival, reproduction, somatic growth, and maturation. The rates or probabilities for these processes can vary by time, by location, and by individual. These processes can co-vary and interact to varying degrees, e.g., an animal can only reproduce when it is in a particular maturation state. Population dynamics models that treat the processes as independent may yield somewhat biased or imprecise parameter estimates, as well as predictions of population abundances or densities. However, commonly used integral projection models (IPMs) typically assume independence across these demographic processes. We examine several approaches for modelling between process dependence in IPMs, and include cases where the processes co-vary as a function of time (temporal variation), co-vary within each individual (individual heterogeneity), and combinations of these (temporal variation and individual heterogeneity). We compare our methods to conventional IPMs, which treat vital rates independent, using simulations and a case study of Soay sheep (Ovis aries). In particular, our results indicate that correlation between vital rates can moderately affect variability of some population-level statistics. Therefore, including such dependent structures is generally advisable when fitting IPMs to ascertain whether or not such between vital rate dependencies exist, which in turn can have subsequent impact on population management or life-history evolution.
\end{abstract}

\section{Hosted file}

main.pdf available at https://authorea.com/users/448090/articles/546961-building-integralprojection-models-with-non-independent-vital-rates 


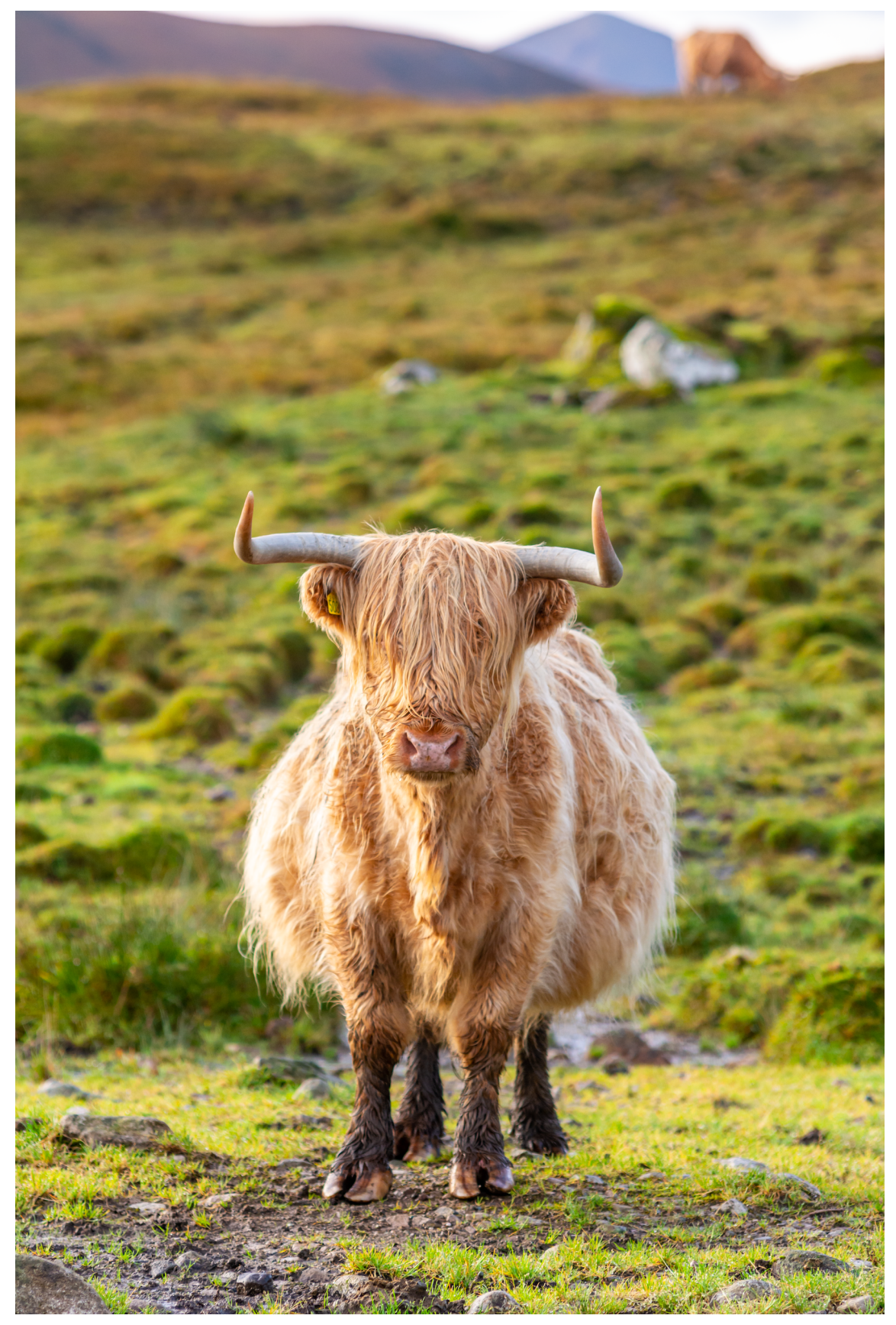

